\title{
Compressed Sensing Dynamic Reconstruction in Rotational Angiography
}

\author{
Hélène Langet ${ }^{1,2,3, \star}$, Cyril Riddell ${ }^{1}$, Yves Trousset $^{1}$, Arthur Tenenhaus ${ }^{2}$, \\ Elisabeth Lahalle ${ }^{2}$, Gilles Fleury ${ }^{2}$, and Nikos Paragios ${ }^{3,4,5}$ \\ 1 GE Healthcare, Interventional Radiology, Buc, France \\ 2 Supélec, SSE department, Gif-sur-Yvette, France \\ 3 ECP, Center for Visual Computing, Châtenay-Malabry, France \\ 4 ENPC, Center for Visual Computing, Champs-sur-Marne, France \\ 5 INRIA Saclay, GALEN Team, Orsay, France
}

\begin{abstract}
This work tackles three-dimensional reconstruction of tomographic acquisitions in $\mathrm{C}$-arm-based rotational angiography. The relatively slow rotation speed of C-arm systems involves motion artifacts that limit the use of three-dimensional imaging in interventional procedures. The main contribution of this paper is a reconstruction algorithm that deals with the temporal variations due to intra-arterial injections. Based on a compressed-sensing approach, we propose a multiple phase reconstruction with spatio-temporal constraints. The algorithm was evaluated by qualitative and quantitative assessment of image quality on both numerical phantom experiments and clinical data from vascular Carm systems. In this latter case, motion artifacts reduction was obtained in spite of the cone-beam geometry, the short-scan acquisition, and the truncated and subsampled data.
\end{abstract}

\section{Introduction}

Rotational angiography provides three-dimensional (3D) qualitative and quantitative information of high clinical interest as the complexity of minimally invasive procedures increases. However, its spread in the clinical practice is limited by the nature of the measurements: 3D reconstruction of C-arm system data is challenging due to the use of cone-beam (CB) geometry, short-scan orbits, as well as truncated and angularly subsampled data. Besides, physiological times (e.g. heart beat, breathing time) are small compared to the gantry rotation speed of $\mathrm{C}$-arm systems. The resulting temporal variations within a scan are so challenging that they are usually not corrected for in the clinical practice.

Temporal variations are addressed through the decomposition of the dynamic $(3 \mathrm{D}+\mathrm{t})$ data into phases where the object can be considered "static" so that $3 \mathrm{D}$ reconstruction is feasible. Each phase defines a subset of projections that must fully sample the volume at this particular time point 9 . To alleviate

\footnotetext{
* Corresponding author: helene.langet@ge.com. This work was supported in part by

ANRT grant number CIFRE-936/2009.
} 
this sampling requirement, additional a priori information must be integrated. Common approaches rely on modeling the motion (e.g. B-splines or dense vector fields) so that each phase can be deduced from a reference one by the motion model. That is a challenging estimation problem though. Motion of the coronary arteries or of an opaque device can be detected in the projective images [1, 8. while breathing motion can been derived from a previous CT acquisition 10. Still, even if a perfect knowledge of the motion reduces the number of unknown volumes to just one, it does not guaranty alone that this unique volume is properly sampled; the acquisition itself must be adapted (e.g. ECG-gating, multiple rotations).

These levels of complexity are not adequate for addressing the need for improving standard C-arm imaging marred by accidental motion artifacts or contrast flow variations. Three-dimensional reconstruction based on compressed sensing (CS) is a new alternative that allows for directly reconstructing subsampled data, and that is applicable even in absence of a motion model, yielding a sparse approximation to the solution through spatial constraints (typically wavelets or total variation filtering) and temporal redundancy $[2,3,5,7,11$. One particularly seducing approach relies on using a temporal constraint based on a prior image equal to the static reconstruction, so that, schematically, motionless areas are determined from the complete data set, while motion-blurred areas are determined from subsampled reconstruction with a total variation constraint [3]. However, with intra-arterial contrast injection, motion blur is not the only degradation present in the standard Feldkamp (FDK) static reconstruction: any part of the image may be degraded by intense streaks due to the presence of inconsistent high-intensity vessel projections in the data, making the static reconstruction a poor prior image. On the positive side, we have shown in [6] in this same context, that an iterative FDK (iFDK) algorithm combined with a spatial constraint called soft background subtraction (SBS) could mitigate subsampling artifacts while preserving the overall aspect of FDK reconstructions, a point of importance for the clinical practice. However, this strategy is not applicable to removing streaks due to incoherent static data and thus cannot provide an improved prior image. We therefore generalize the approach presented in [6] to the reconstruction of a series of volumes based on combining the SBS spatial constraint to a temporal constraint that enforces the sparsity of the difference between time points. We show how this multiple-phase reconstruction with spatio-temporal constraints can be casted in the same mathematical framework of an iFDK algorithm associated to proximal splitting schemes. It is applied to two types of temporal inconsistencies within injected vessels: small displacements induced by the blood flow pulsatility and contrast flow variability during the scan due to delayed opacification.

\section{Dynamic Reconstruction}

Let us consider $3 \mathrm{D}+\mathrm{t}$ data with $M$ phases. We denote $f$ and $p$ the vectors that respectively contain the imaged volume at different time points $f_{i}=f\left(t_{i}\right)$ and 
the data, where $p_{i}$ refers to the $2 \mathrm{D}$ projection subset assigned to the volume $f_{i} . R$ is the block-diagonal matrix that models the rotational $\mathrm{CB}$ acquisition process.

$$
f=\left(\begin{array}{c}
f_{1}=f\left(t_{1}\right) \\
\vdots \\
f_{M}=f\left(t_{M}\right)
\end{array}\right) \quad p=\left(\begin{array}{c}
p_{1} \\
\vdots \\
p_{M}
\end{array}\right) \quad R=\left(\begin{array}{ccc}
R_{1} & & \\
& \ddots & \\
& & R_{M}
\end{array}\right)
$$

The noise-free tomographic problem consists in solving the system of linear equations

$$
R f=p .
$$

This problem is underdetermined because the set of measurements $p$ is small with respect to $f$.

\subsection{Compressed Sensing Phase Reconstruction with Spatial Constraint (CS-Ps)}

For the case of angiographic data that contain sparse intense vessels over a nonsparse background, we presented in [6] a CS-based reconstruction strategy, here called CS-Ps, that enables the efficient removal of vessel-induced subsampling artifacts. CS-Ps consists in solving a sequence of $\mathrm{N} \ell_{1}$-regularized problems indexed by a set of decreasing regularization hyperparameters: $\Lambda_{s}=\left\{\lambda_{s}^{(n)} \mid n=\right.$ $1, \cdots, N\}$, such that $\lambda_{s}^{(1)} \geq \cdots \geq \lambda_{s}^{(N)}$. Each problem has the form:

$$
\underset{f}{\operatorname{argmin}}\left[Q(f)+\chi_{\lambda_{s}^{(n)}}(f)\right] \text {, }
$$

where $Q(f)=\frac{1}{2}(R f-p)^{T} D(R f-p)$ denotes the $\ell_{2}$-data fitting term, ${ }^{T}$ the transpose symbol, $D$ the ramp filter that is positive and diagonal in the Fourier domain, and $\chi_{\lambda_{s}^{(n)}}(f)=\lambda_{s}^{(n)} \Phi_{s}(f)=\lambda_{s}^{(n)}\|f\|_{1}^{+}$the combination of the image $\ell_{1}$-norm with positivity. Problem (2) is solved with proximal operators using a splitting scheme [4]:

$$
\left\{\begin{aligned}
f^{\left(k+\frac{1}{2}\right)} & =f^{(k)}-\tau \nabla Q\left(f^{(k)}\right)=f^{(k)}+\tau R^{T} D\left(p-R f^{(k)}\right) \\
f^{(k+1)} & =\operatorname{prox}_{\tau \chi_{\lambda_{s}(n)}}\left(f^{\left(k+\frac{1}{2}\right)}\right) \equiv \underset{g}{\operatorname{argmin}}\left[\tau \chi_{\lambda_{s}^{(n)}}(g)+\frac{1}{2}\left\|g-f^{\left(k+\frac{1}{2}\right)}\right\|_{2}^{2}\right],
\end{aligned}\right.
$$

where $\tau$ is a standard gradient descent step. The proximal operator $\operatorname{prox}_{\tau \chi_{\lambda_{s}(n)}}$ defined in (3) has a direct expression as the SBS operator: it soft-thresholds the positive values by $\tau \lambda_{s}^{(n)}$ and sets the negative ones to zero. The minimization at $\lambda_{s}^{(n)}$ identifies a sparse approximation that best fits the data with a level of sparsity that is proportional to $\lambda_{s}^{(n)}$. It is used as a warm-start to the minimization at $\lambda_{s}^{(n+1)}$. In practice, iteration (3) results in a standard FDK reconstruction scaled by $\tau$ and segmented with threshold $\tau \lambda_{s}^{(n)}$. Thus, CS-Ps consists in reconstructing high-intensity vessels first, without streaks, while the background is 
progressively reintroduced as $\lambda_{s}^{(n)}$ tends to zero. The recent work [12] identifies this process as an approximate homotopy continuation strategy whose convergence is proved given that each problem is solved with sufficient precision and $\lambda_{s}^{(n)}$ is geometrically decreased. With iterative FDK, we observed convergence when solving each problem with one iteration and using a simple linear decrease.

\subsection{Compressed Sensing Phase Reconstruction with Spatio-Temporal Constraint (CS-Pst)}

CS-Ps reconstructs each phase independently so that the sampling of the static background structures is significantly reduced. To recover full sampling of the background we have to take inter-phase correlations into account. Since we focus on the vessel temporal variations only, it is relevant to assume sparsity of the difference to the weighted mean $\Phi_{t}(f)=\sum_{i=1}^{M}\left\|f_{i}-\frac{1}{M} \sum_{j=1}^{M} \omega_{j} f_{j}\right\|_{1}$ where $\omega_{j}$ accounts for the possible difference in the phase subset cardinality. Merging each phase background is relevant only if it does not contain vessel-induced streaks. This suggests extending the sparsity constraint of problem (2) with a temporal constraint $\Phi_{t}$ of hyperparameter $\lambda_{t}$ such that:

$$
\chi_{\lambda_{s}^{(n)}}(f)=\lambda_{s}^{(n)} \Phi_{s}(f)+\lambda_{t} \Phi_{t}(f) .
$$

Here, hyperparameter $\lambda_{s}$ is handled exactly as in CS-Ps because the background is not assumed sparse, while hyperparameter $\lambda_{t}$ can be assigned a fixed value without introducing a bias because temporal sparsity can be assumed. We denote CS-Pst this approach. Alternatively, the weighted mean can be replaced by a prior image $f_{P}$, leading to $\Phi_{t}(f)=\sum_{i=1}^{M}\left\|f_{i}-f_{P}\right\|_{1}$. We denote CS-Psp this latter constraint (4) with the prior image chosen equal to the static reconstruction as in [3].

Application of $\operatorname{prox}_{\tau \chi_{\lambda_{s}^{(n)}}}$ means that all constraints are satisfied at once. In absence of a direct expression for $\operatorname{prox}_{\tau \chi_{\lambda_{s}(n)}}$, we turn to the Dykstra-like proximal algorithm to iteratively compute $\operatorname{prox}_{\tau \chi_{\lambda_{s}(n)}}(f)$ using $\operatorname{prox}_{\lambda_{s}^{(n)} \Phi_{s}}$ and $\operatorname{prox}_{\lambda_{t} \Phi_{t}}$. The Dykstra-like scheme is detailed in Sec. 5 of [4] (p.10), and is here nested within scheme (2). The direct expression of $\operatorname{prox}_{\lambda_{t} \Phi_{t}}$ is obtained by rewriting the temporal constraint as $\Phi_{t}(f)=\left\|H_{t}\left(f-f_{P}\right)\right\|_{1}$, where $H_{t}$ is an invertible matrix. For the difference to the weighted mean, $f_{P}=0$ and $H_{t}$ transforms the temporal intensity at a given voxel into an average component and differential components that are thresholded. In the case of the prior image, $H_{t}$ is set equal to the identity matrix.

\section{Evaluation}

In order to establish the reconstruction performances of CS-Pst and CS-Psp with respect to CS-Ps, we built a numerical phantom with three phases by adding simulated injected arteries (from 1500 to $6000 \mathrm{HU}$ ) to a $512 \times 512$ abdominal CT crosssection where background structures are valued between 1000 and 2000 HU. Note 
that we consider positive HU unit, where air is $0 \mathrm{HU}$ and water is $1000 \mathrm{HU}$. We simulated the acquisition in parallel geometry of data with (a) a pulsatile motion synchronized with an ECG signal, and (b) a motion where each phase is defined by a subset of contiguous projections, resulting in a limited-angle sampling. Acquisition settings were chosen to fit clinical routine, where C-arm systems record projections at 30 frames $/ \mathrm{s}$ during an approximately $200^{\circ}$ rotation at $40^{\circ} / \mathrm{s}$ delivering about 150 projections in total. Reconstruction settings are as follows: $\tau=0.9, \mathrm{~N}$ $=200$ thresholds, $\lambda_{s}^{(1)}=3000 \mathrm{HU}$ and is linearly decreased to $\lambda_{s}^{(N)}=0 \mathrm{HU}$ for CS-Ps, CS-Pst and CS-Psp, and $\lambda_{t}=50 \mathrm{HU}$ for CS-Pst and CS-Psp.

Figure 1 gives comparative assessment of the reconstruction quality. Figure. 1.(a) displays two regions of interest (RoI) of the phantom: with static structures only (top image) and with dynamic injected vessels over static background for two phases (middle and bottom images). CS-Ps reconstruction is shown in Fig. 11.(b) to restore the temporal resolution of the vessels without introducing subsampling streaks, but the background is poorly depicted due to the substantial subsampling of each phase. Comparing to Fig. 1. (c), the prior image constraint of CS-Psp improved the background, while preserving the temporal resolution. A strong streak pattern remains in all RoIs however, that is a slightly attenuated version of the one present in the prior image (image not shown). The best restoration is thus obtained by CS-Pst as shown in Fig. 1.(d). CS-Pst results for the limited-angle case are presented in 1. (e). It outperformed CS-Ps and CSPsp in the same manner (images not shown). We quantified streak intensity by measuring the maximum intensity which is not a vessel in the RoI with dynamic injected vessels: for the pulsatile case streak intensity decreased from $1872 \mathrm{HU}$ for the prior image to $1636 \mathrm{HU}$ and $1302 \mathrm{HU}$ for CS-Psp and CS-Pst respectively; for the limited-angle case it decreased from $1954 \mathrm{HU}$ to $1629 \mathrm{HU}$ and $1406 \mathrm{HU}$.

The convergence behavior was monitored at each iteration by the normalized root mean square deviation $d_{r}=\sqrt{\frac{\sum_{j=1}^{J_{r}}\left(f_{i, j}-\tilde{f}_{i, j}\right)^{2}}{\sum_{j=1}^{J_{r}} \tilde{f}_{i, j}^{2}}}$ where $J_{r}$ is the number of pixels in RoI $r$ and $\tilde{f}_{i}$ is the phantom. Figure 2 contains the plots of $\log d_{r}$ with respect to the number of iterations for two RoIs: one covering the area with moving vessels displayed in the the middle row of Fig. 1 and shown in graph 2. (a), the other covering the background displayed in the top row of Fig. 1 and shown in graph 2.(b). Discrepancy between the prior image and the reference image over each RoI is shown as a black dashed line. The final deviations of graph 2. (a) reflect the improved recovery of the moving structures with all CS dynamic reconstructions. In graph 2,(b), CS-Ps high deviation reflects poor background recovery. For CS-Psp, background recovery nears the level of the prior image, but cannot improve it by construction. On the contrary, CS-Pst restores the background below the discrepancy level of the prior image.

Results on real data are analyzed in Fig. 3 where standard clinical (i.e. static) reconstruction is compared to CS-Pst. The first dataset is an exam of the renal arteries, in which small displacements of the catheter occur due to the pulsatile blood flow. We manually splitted the scan into four phases, relying on the vertical translation of the tip, that is visible on the $2 \mathrm{D}$ projections. The resulting 


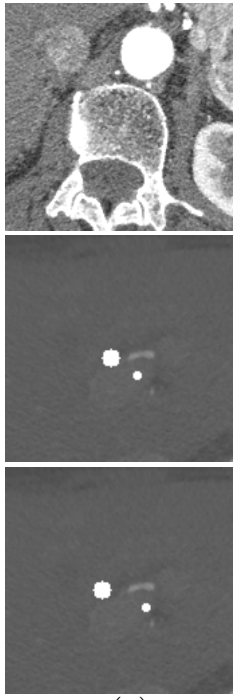

(a)
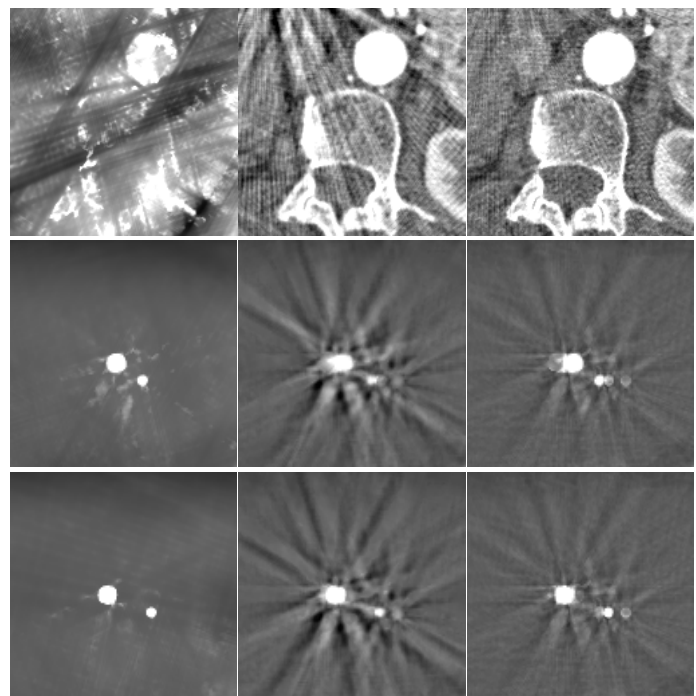

(b)

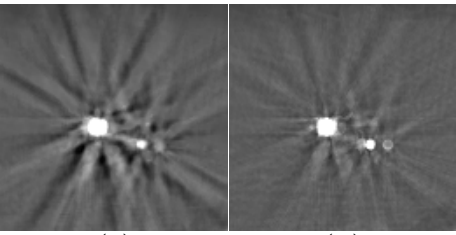

(c)

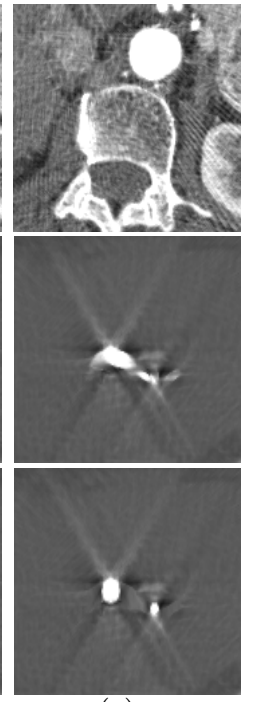

(e)

Fig. 1. Simulated data. First row: RoI with static structures - HU display range: 800 to 1400; second and third rows: RoI with dynamic injected vessels over static background for phase 1 and phase 2 respectively - HU display range: 100 to 2800. (a) Ground-truth. Pulsatile case: (b) CS-Ps; (c) CS-Psp; (d) CS-Pst. Limited-angle case: (e) CS-Pst.

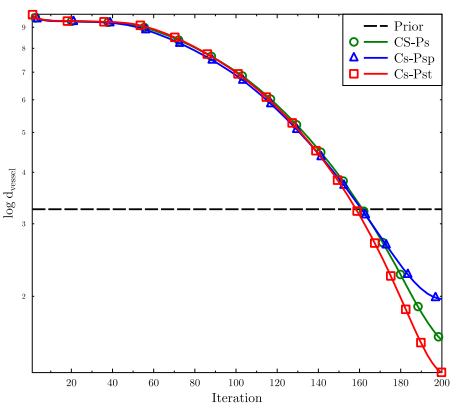

(a)

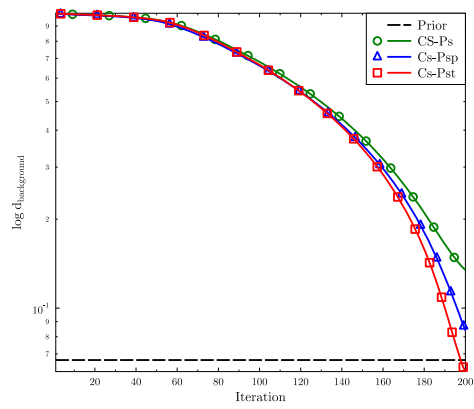

(b)

Fig. 2. Convergence curves for the numerical phantom with pulsatile motion: (a) RoI with dynamic injected vessels over static background for phase 1; (b) RoI with static structures

phases contained $33,36,40$, and 38 projections respectively. The second dataset is an exam of the cerebral vessels. During the first half of the scan the right vertebral artery (RVA, most left vessel in the axial slices in Fig. 3.(2)) did not appear opacified, while the left vertebral artery (LVA, most right vessel) was seen fully opacified during the whole scan. We thus splitted the scan into two 
phases that contained 72 and 75 projections respectively. Static reconstructions integrate all temporal variations: the position of the tip is blurred in the first exam (Fig. 3. (1a)), while the intensity of the RVA peak is averaged to $9130 \mathrm{HU}$ (Fig. 3. (2a)), that is less than $60 \%$ of the LVA peak (15310 HU). In addition, intense streak artifacts degrade the background: the displacements of the catheter yield a rotating pattern of positive and negative streaks in the first case, while the lack of opacification in the lateral projections yields horizontal and vertical negative streaks in the second case. CS-Pst reconstruction recovered some temporal resolution since temporal variations are visible in the reconstructed phases, even though our phase selection was approximate. This is particularly striking when looking at the associated maximum intensity projection (MIP) given in the second row of Fig. 3, the vertical translation of the catheter is well visible (Fig. 3. (1b) and (1c)); the RVA is only visible during phase 2, while the LVA is visible in both phases (Fig. 3. (2b) and (2c)). In terms of quantification the RVA peak was measured to be $1960 \mathrm{HU}$ for phase 1 and $12290 \mathrm{HU}$ for phase 2, while the LVA peak was measured to be around $13600 \mathrm{HU}$ for both phases. As for the background CS-Pst reduced the streaks located close to the catheter tip (Fig. 3. (1b) and (1c)), and nearly eliminated the horizontal and vertical streaks of the RVA (Fig. 3. (2b) and (2c)).

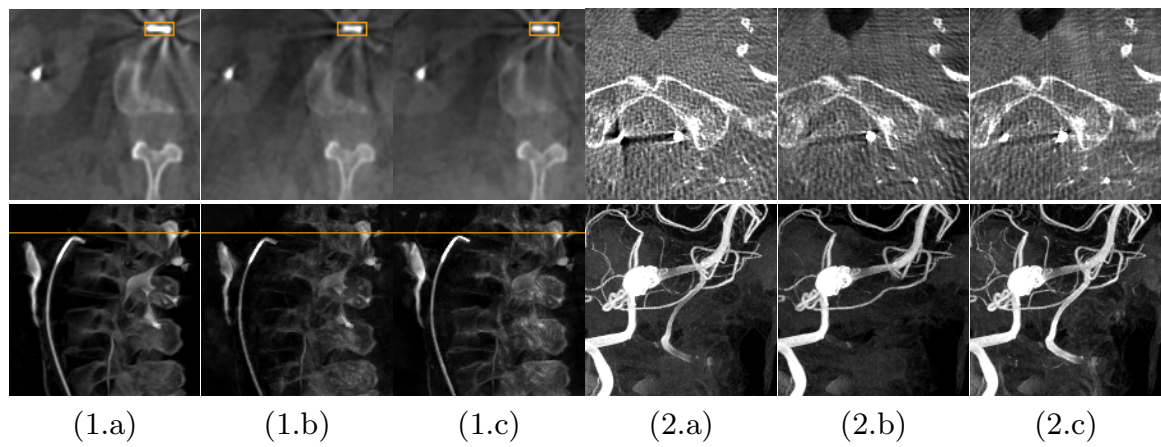

Fig. 3. Clinical data. (1) Pulsatile case; (2) Limited-angle case. First row: axial slice detail - HU display range: (1) 350 to 1550 (2) 0 to 2400. Second row: MIP detail - HU display range: (1) 1100 to 4400 (2) 1000 to 9500. Algorithms: (a) static reconstruction; CS-Pst for (b) phase 1 and (c) phase 2.

\section{Conclusion and Discussion}

We proposed a $3 \mathrm{D}+\mathrm{t}$ reconstruction that relies on a CS approach with spatiotemporal constraints. The evaluation of the algorithm with numerical experiments and two typical angiographic datasets demonstrated qualitative and quantitative improvements. These results were obtained in a clinical context with limited assumptions: high-intensity sparse structures but non-sparse background, temporal correlation but no motion modeling, only manual phase selection. For an actual clinical usage, automatic phase selection is a requirement. It 
is challenging, but to a lesser degree than motion estimation and modeling. Future work will aim at applying the proposed mathematical framework to a larger class of problems because it relies on proximal operators. If applicable, stronger spatial sparsity can be enforced through wavelets or total variation filters, which are proximal operators. Another direction is to strengthen the proposed temporal constraint by computing a mean image that would account for some (i.e. even incomplete) inter-phase registration based upon a priori motion knowledge. This constraint could still be applied through a proximal operator similar to the one presented here. In order to simplify the presentation, the temporal constraint was also set equal for all voxels. This is not a requirement: in the case of a delayed opacification, each voxel could be treated independently, with its own phase selection and associated temporal constraint and proximal operator.

\section{References}

1. Blondel, C., Malandain, G., Vaillant, R., Ayache, N.: Reconstruction of coronary arteries from a single rotational X-ray projection sequence. IEEE Transactions on Medical Imaging 25(5), 653-663 (2006)

2. Candès, E., Romberg, J., Tao, T.: Robust Uncertainty Principles: Exact Signal Reconstruction from Highly Incomplete Frequency Information. IEEE Transactions on Information Theory 52(2), 489-509 (2006)

3. Chen, G.H., Tang, J., Leng, S.: Prior Image Constrained Compressed Sensing (PICCS). Medical Physics 35(2), 660-663 (2008)

4. Combettes, P.L., Pesquet, J.C.: Proximal Splitting Methods in Signal Processing. In: Fixed-Point Algorithms for Inverse Problems in Science and Engineering, ch. 10, vol. 49, pp. 185-212 (2011)

5. Jia, X., Lou, Y., Dong, B., Tian, Z., Jiang, S.: 4D Computed Tomography Reconstruction from Few-Projection Data via Temporal Non-local Regularization. In: Jiang, T., Navab, N., Pluim, J.P.W., Viergever, M.A. (eds.) MICCAI 2010, Part I. LNCS, vol. 6361, pp. 143-150. Springer, Heidelberg (2010)

6. Langet, H., Riddell, C., Trousset, Y., Tenenhaus, A., Lahalle, E., Fleury, G., Paragios, N.: Compressed Sensing Based 3D Tomographic Reconstruction for Rotational Angiography. In: Fichtinger, G., Martel, A., Peters, T. (eds.) MICCAI 2011, Part I. LNCS, vol. 6891, pp. 97-104. Springer, Heidelberg (2011)

7. Lustig, M.: Sparse MRI. Ph.D. thesis, Stanford University (September 2008)

8. Perrenot, B., Vaillant, R., Prost, R., Finet, G., Douek, P., Peyrin, F.: Motion correction for coronary stent reconstruction from rotational x-ray projection sequences. IEEE Trans. Med. Imaging 26(10), 1412-1423 (2007)

9. Prümmer, M., Hornegger, J., Lauritsch, G., Wigström, L., Girard-Hughes, E., Fahrig, R.: Cardiac C-Arm CT: A Unified Framework for Motion Estimation and Dynamic CT. IEEE Trans. Med. Imaging 28(11), 1836-1849 (2009)

10. Rit, S., Sarrut, D., Desbat, L.: Comparison of analytic and algebraic methods for motion-compensated cone-beam ct reconstruction of the thorax. IEEE Trans. Med. Imaging 28(10), 1513-1525 (2009)

11. Sidky, E.Y., Duchin, Y., Pan, X., Ullberg, C.: A constrained, total-variation minimization algorithm for low-intensity x-ray CT. Medical Physics 38(S1), S117-S125 (2011)

12. Xiao, L., Zhang, T.: A Proximal-Gradient Homotopy Method for the Sparse LeastSquares Problem. CRR abs/1203.3002 (2012) 